\title{
Electropalatographic, acoustic, and perceptual data on adaptation to a palatal perturbation
}

Wendi A. Aasland, Shari R. Baum, and David H. McFarland

Citation: The Journal of the Acoustical Society of America 119, 2372 (2006);

View online: https://doi.org/10.1121/1.2173520

View Table of Contents: http://asa.scitation.org/toc/jas/119/4

Published by the Acoustical Society of America

\section{Articles you may be interested in}

An electropalatography study of nasal-trill/lateral sequences in Spanish

The Journal of the Acoustical Society of America 133, 3608 (2013); 10.1121/1.4806709

On the relationship between palate shape and articulatory behavior

The Journal of the Acoustical Society of America 125, 3936 (2009); 10.1121/1.3125313

Dynamic electropalatography

The Journal of the Acoustical Society of America 93, 2416 (1998); 10.1121/1.405914 


\title{
Electropalatographic, acoustic, and perceptual data on adaptation to a palatal perturbation
}

\author{
Wendi A. Aasland and Shari R. Baum ${ }^{\text {a) }}$ \\ School of Communication Sciences \& Disorders, McGill University, Montreal, QC H3G 1A8, Canada \\ David H. McFarland \\ Ecole d'Orthophonie et d'Audiologie, Université de Montréal, Montreal, QC H3C 3J7, Canada
}

(Received 6 September 2005; revised 17 January 2006; accepted 17 January 2006)

\begin{abstract}
Exploring the compensatory responses of the speech production system to perturbation has provided valuable insights into speech motor control. The present experiment was conducted to examine compensation for one such perturbation - a palatal perturbation in the production of the fricative /s/. Subjects wore a specially designed electropalatographic (EPG) appliance with a buildup of acrylic over the alveolar ridge as well as a normal EPG palate. In this way, compensatory tongue positioning could be assessed during a period of target specific and intense practice and compared to nonperturbed conditions. Electropalatographic, acoustic, and perceptual analyses of productions of /asa/ elicited from nine speakers over the course of a one-hour practice period were conducted. Acoustic and perceptual results confirmed earlier findings, which showed improvement in production with a thick artificial palate in place over the practice period; the EPG data showed overall increased maximum contact as well as increased medial and posterior contact for speakers with the thick palate in place, but little change over time. Negative aftereffects were observed in the productions with the thin palate, indicating recalibration of sensorimotor processes in the face of the oral-articulatory perturbation. Findings are discussed with regard to the nature of adaptive articulatory skills. (c) 2006 Acoustical Society of America. [DOI: 10.1121/1.2173520]
\end{abstract}

PACS number(s): 43.70.Ep, 43.70.Bk [BHS]

Pages: 2372-2381

\section{INTRODUCTION}

Exploring the compensatory responses of the speech production system to a variety of static and dynamic perturbations has provided valuable insights into a variety of fundamental processes, including central or predictive representations of speech movements and their interaction with sensory feedback (Löfqvist, 1997; Tremblay, Shiller, and Ostry, 2003). For the oral motor system, an adaptive response paradigm may provide an effective means of exploring many fundamental aspects of speech production, including the development of novel articulatory programs related to speech motor learning. We have completed a series of investigations designed to measure the adaptive responses of the speech motor control system to a variety of perturbations to oral form and function (Baum and McFarland, 2000; Baum, McFarland, and Diab, 1996; Baum and McFarland, 1997; McFarland, Baum, and Chabot, 1996). To induce a structural perturbation, we have employed an artificial palate with a substantial buildup of acrylic over the alveolar ridge, similar to that used initially by Hamlet and colleagues (Hamlet, Cullison, and Stone, 1979) and further modified for our experimental purposes (Baum and McFarland, 1997; McFarland, Baum, and Chabot, 1996). This artificial palate, when contrasted with one with a very thin acrylic covering and

\footnotetext{
a) Author to whom correspondence should be addressed: Shari R. Baum, Ph.D., School of Communication Sciences \& Disorders, McGill University, 1266 Pine Avenue West, Montreal, Quebec H3G 1A8, Canada. Electronic mail: shari.baum@mcgill.ca
}

with no palate in place, significantly perturbs consonant production (in particular /s/ production) and may involve a rather lengthy adaptation period for normal articulatory profiles to be developed. Target intensive practice was found to accelerate the adaptation and make it reasonable to study compensatory processes within a short period of time (e.g., one hour) (Baum and McFarland, 1997). Results revealed significant improvements in /s/ production during the course of the adaptation period, with evidence of the formation of novel articulatory programs and sensorimotor recalibration of the articulatory space. Negative aftereffects were also observed, in which the accuracy of speech produced without the palate in place as well as with the thin appliance, was negatively impacted as a result of the target intensive practice with the modified palatal contour (Baum and McFarland, 1997).

These earlier investigations employed both acoustic and perceptual measures to make judgments about speech compensation and articulatory modification in response to oral articulatory perturbation. What was lacking was information regarding potential compensatory movements and contacts of the speech articulators. Such information is crucial for understanding motor control strategies related to the adaptation process. We have recently developed (Aasland, Baum, and McFarland, 2004a; b) a new technological modification of an existing electropalatographic system that allows for the tongue movement contact electrodes to be embedded in the modified palatal contour (Hamlet, Stone, and McCarty, 1978). In this way, compensatory tongue positioning can be recorded simultaneously with speech acoustics during the 
course of adaptation to a significant perturbation to oral form. Such compensatory responses can be compared to standard EPG appliances that involve a very thin layer of acrylic that has been shown to be relatively less perturbing to speech production than the thick palatal appliance (Hamlet, Cullison, and Stone, 1979).

Electropalatography (EPG) is an extremely wellestablished measurement process that has been used both for understanding fundamental aspects of speech production and for clinical intervention to encourage appropriate tongue positioning for a variety of speech disorders, such as those associated with traumatic brain injury, velopharyngeal inadequacy/cleft palate, articulation disorders, deafness, and other communicative disorders (e.g., Flege, Fletcher, and Homiedan, 1988; Fletcher and Newman, 1991; Fletcher, McCutcheon, and Wolf, 1975; Gibbon, 2004; Gibbon and Wood, 2003; Goozée, Murdoch, and Theodoros, 2003; Parsloe, 1998). Tongue-palate contact patterns reflecting normal consonant and vowel articulation have been relatively well described using this technique and some studies have combined EPG analyses with more dynamic movement assessments such as by ultrasound (Stone, Faber, Raphael, and Shawker, 1992). Consequently, correlative data exist that allow for the referral of tongue-palate contact patterns to those related to tongue shape and cross-sectional area during speech sound production. The fricative /s/ (the target consonant of the current investigation) is articulated with an anterior tongue constriction and concave central groove that directs the airstream turbulence against the upper or lower incisors (Fletcher and Newman, 1991; Narayanan, Alwan, and Haker, 1995; Stevens, 1998; Stone et al., 1992). Marginal lateral tongue contact and upward force vectors form a "lingualpalatal brace" for central groove configuration. Groove depth averages $5.6 \mathrm{~mm}$ but is highly speaker dependent (Stone et al., 1992), as is the cross-sectional tongue configuration at the point of constriction, which varies from slit-like to more circular (Narayanan et al., 1995). Midsagittal tongue shape is overall convex to concave, running anteriorly to posteriorly, but is also speaker dependent and is related to apical versus laminal articulation (Narayanan et al., 1995).

The present investigation was designed to use the EPG measurement tool and these previous detailed analyses of normal fricative production to explore speech compensatory processes related to the presence of oral articulatory perturbations. Tongue-palate contact information was combined with detailed acoustic and perceptual measures (as in previous investigations) to provide a relatively comprehensive account of compensatory behaviors and potential individual differences in such behaviors. The overall goal is to understand speech motor control processes underlying the development and refinement of normal speech production. Such information is necessary for an increased understanding of a number of speech disorders and their potential remediation.

\section{METHODS}

\section{A. Production experiment}

\section{Subjects}

Nine young adult (19-27 years) native speakers of English served as participants. All reported a negative history of

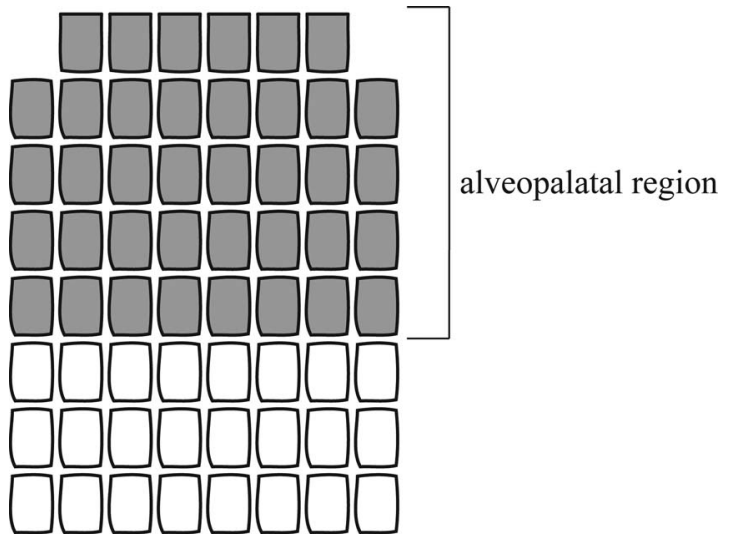

FIG. 1. Display of electrode array with the shaded portion illustrating the alveopalatal region. The anterior of the palate is at the top of the figure.

speech, language, and hearing impairments and none had any prior experience wearing a dental appliance. Subjects were screened for normal dental occlusion at the time of testing.

\section{Stimuli and procedures}

Two EPG artificial palates were constructed for each participant—one "normal" EPG palate, approximately $1 \mathrm{~mm}$ thick throughout ("thin" palate), with an array of 62 electrodes in keeping with the layout prescribed for Articulate Assistant EPG software (Wrench, 2003), and one "perturbed" EPG palate with a $6 \mathrm{~mm}$ buildup of acrylic at the alveolar ridge ("thick" palate), maintaining the same spatial distribution of the electrodes. These thick and thin ${ }^{1}$ palates were identical in dimensions to those used in our previous investigations of compensatory responses to oral-articulatory perturbation and the reader is referred to these earlier studies for the artificial palate configurations (e.g., McFarland and Baum, 1995). The array includes seven rows of eight electrodes each, with the exception of the anterior-most row, which contains only six electrodes (see Fig. 1). Regions defining alveolar, palatal, and lateral borders are illustrated in the figure.

To assess the impact of the perturbations on compensatory tongue positioning, speakers were required to produce multiple repetitions of the bisyllable /asa/ under a variety of experimental conditions and at five time intervals. The target measured in each instance was the medial /s/. The target stimulus was first elicited ${ }^{2}$ ten times with no palate in place to provide a baseline of normal /s/ production. Subsequently, ten repetitions of /asa/ were produced first with the thin palate in place, followed by ten repetitions with the thick palate in place. This entire procedure was then repeated for a total of 20 exemplars in each condition at Time 0 . As in our previous investigations, stimuli were elicited every $15 \mathrm{~min}$ for a period of one hour during which speakers read /s/-laden passages with the thick palate in place (see, e.g., Baum and McFarland, 1997). At each 15 min interval (Times 15, 30, $45,60)$, two blocks of ten repetitions of /asa/ were elicited with the thick palate in place, alternating with two blocks of ten repetitions with the thin palate in place. After Time 0 , productions were elicited first with the thick palate, which was already in place, to ensure that speakers maintained any 
compensatory adjustments developed during the practice interval. At Time 60, an additional two blocks of ten repetitions of /asa/ were elicited with no palate in place to examine any potential aftereffects of the $1 \mathrm{~h}$ palatal modification. Stimuli were recorded directly onto the computer using the Articulate Assistant software (Wrench, 2003) to ensure synchronization of the acoustic and EPG signals.

\section{Acoustic analyses}

Following earlier studies (Baum and McFarland, 1997; McFarland et al., 1996), we determined three acoustic characteristics: (1) /s/ duration, measured from the onset to the offset of visible frication noise in the waveform display; (2) centroid frequency (or the first moment of the spectral distribution) at the /s/ midpoint, representing a weighted average of spectral peak frequencies; and (3) standard deviation of the spectrum (or the second moment of the spectral distribution).

\section{EPG analyses}

The goal of the EPG analyses was to provide information of compensatory tongue contact positioning in the face of oral-articulatory perturbation during /s/ production. Measures were based on previous assessments of positioning during normal and disordered articulation and were modified for the current experimental context. We began with a very large number of EPG contact measures computed at various points and in various palatal regions. As the majority of these measures were quite comparable, we report here only measures based on contact across the entire palate at the (temporal) point of maximum contact during /s/ production, as well as contact measures within the alveopalatal region at the point of maximum contact (when they differ from those for the whole palate). These two regions of interest were found to capture compensatory tongue positioning.

A measure of whole contact was computed, reflecting the percentage of sensors contacted averaged across all time frames within the maximum contact region. Similarly, a measure of alveopalatal contact was calculated, reflecting the percentage of sensors contacted within the five anterior-most rows of electrodes. A variability index, designed to reflect the stability of articulatory gestures, was calculated by computing the percent frequency of activation of each contact across relevant frames. Activation frequencies of $0 \%$ or $100 \%$ reflect stability and are associated with an index of 0 ; as contact frequency approaches 50\% (i.e., highly inconsistent), a maximum variability index of 50 is assigned. A center of gravity index was also computed, indicating whether primary contact is more anterior or more posterior across the whole palate. Finally, a laterality index was computed, reflecting the degree to which average contact was more toward the midline or the outer sides of the palate; higher values indicate more lateral contact and provide an indication of groove width for $/ \mathrm{s} /$.

\section{B. Perception experiment \\ 1. Subjects}

The listeners were ten native speakers of English (aged 20-29) who were phonetically naïve and exhibited no history of speech, language, or hearing impairment. None of the listeners had participated as speakers in Experiment 1.

\section{Stimuli and procedure}

Recordings from five speakers chosen at random from the production experiment served as stimuli for the perception experiment. (Use of the entire stimulus set from all speakers would have rendered the experiment too long.) For each speaker, a single clear production ${ }^{3}$ of /asa/ was selected from those stimuli originally produced with no palate in place at Time 0 (i.e., before the start of the experimental manipulations) to serve as a "typical" exemplar. These typical exemplars were used as a point of comparison against which listeners were asked to rate the quality of /asa/ productions from the thin and thick palate conditions, as well as those from the no-palate condition recorded at Time 60.

The 90 tokens from each of the 5 speakers were included as follows: 10 tokens each (selected at random) from the thin and thick palate conditions at Times $0,15,30,45$, and 60, as well as 10 tokens from the no-palate condition at Time 60 (as noted above). Listeners were prompted prior to each trial with a visual "get ready" cue on a computer monitor, followed by a 100 silent interval. The typical exemplar for the individual speaker was then presented over closed headphones, followed after $750 \mathrm{~ms}$ by an experimental stimulus from that speaker. Listeners were instructed to rate the second stimulus, focusing solely on the $/ \mathrm{s} /$, on a scale from " 1 " (very poor quality /s/) to " 5 " (very good quality /s/, equivalent to typical exemplar). Five practice trials representing variations in stimulus quality (as judged by the experimenters) preceded the experiment. If the listener appeared not to have understood the instructions on the basis of results of the practice trials, instructions were repeated and the practice stimuli rerun. Trials were presented in a fixed random order, divided into three blocks to permit two breaks during the $45 \mathrm{~min}$ perception experiment.

\section{RESULTS}

\section{A. Acoustic analyses}

Mean centroid frequencies (collapsed across speakers) computed at each time period in the thin and thick palate conditions, as well as those calculated for productions with no palate in place at Times 0 and 60, are displayed in Fig. 2. As may be observed from the graph, upon first insertion of either palate, but notably the thick palate, centroid frequencies are substantially lower than in the no palate condition. However, within 15 min of practice with the thick palate in place, that pattern is reversed for the thick palate and remains that way throughout the practice period. These observations are confirmed by a Time $(\mathrm{T} 0, \mathrm{~T} 15, \mathrm{~T} 30, \mathrm{~T} 45, \mathrm{~T} 60) \times$ Palate $(N, P)$ ANOVA, which yielded main effects of Time $[F(4,32)=5.195, p<0.002]$ and Palate $[F(1,8)=9.288, p$ $<0.02]$, and a Time $\times$ Palate interaction $[F(4,32)$ 


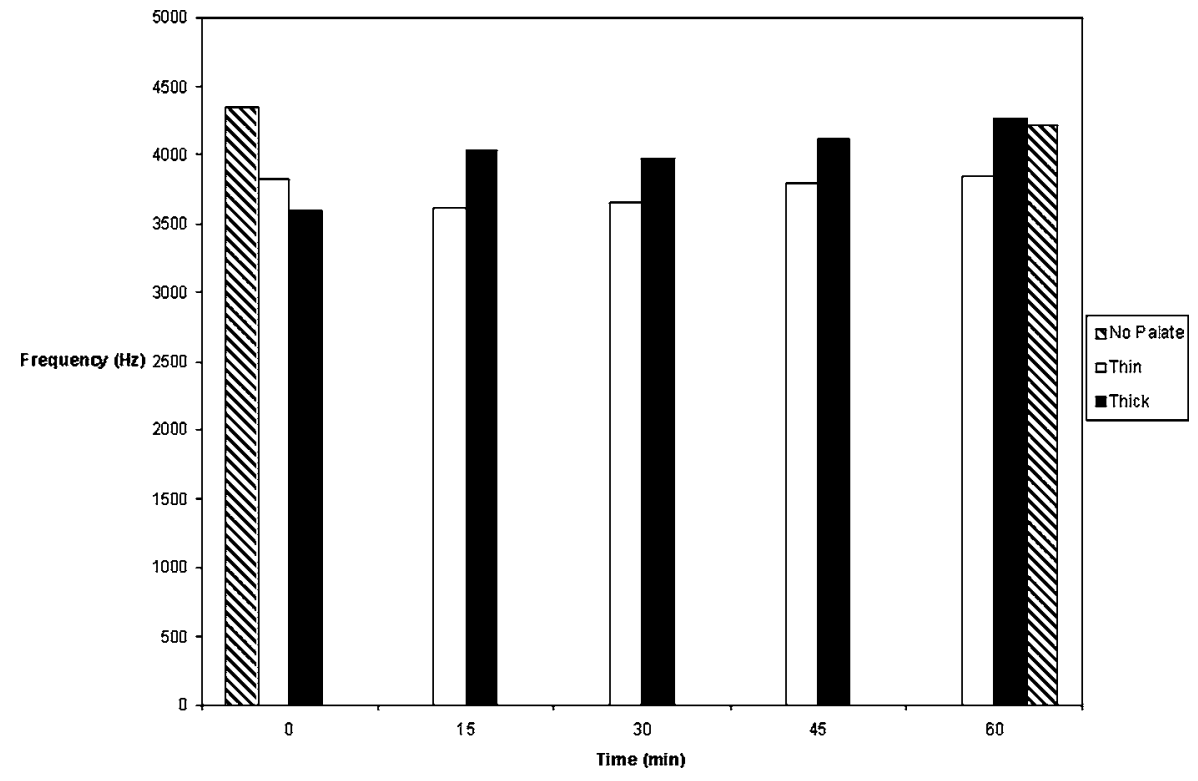

FIG. 2. Mean centroid frequencies over all time periods for the thin and thick palate conditions, and at times 0 and $60 \mathrm{~min}$ for the no-palate (NP) condition.
$=16.495, p<0.001]$. Post hoc analysis of the interaction using the Newman-Keuls procedure $(p<0.05)$ confirmed that at Time 0 , centroids in the thin condition were significantly higher than in the thick condition, whereas at all other time periods, centroids in the thick condition were significantly higher than in the thin condition. Comparing within conditions over time, centroids in the thin condition were significantly higher at Time 0 relative to Times 15 and 30 only. Within the thick palate condition, centroids at Time 0 were significantly lower than at Times 15, 30, and 45, which did not differ from one another; centroids at Time 60 were significantly higher than at all other times.

A separate ANOVA examined centroid frequencies in the no-palate and thin and thick conditions at Times 0 and 60 only. This Time $(0,60) \times$ Palate (no palate, thin, thick) ANOVA revealed a significant main effect of Palate $[F(2,16)=16.734, p<0.001]$ and a Time $\times$ Palate interaction $[F(2,16)=14.793, p<0.001]$. Post hoc analysis of the interaction yielded significant differences between the no-palate and both thin and thick palate conditions at Time 0 ; the thin and thick conditions did not differ from one another. In contrast, at Time 60 , centroids in the thin condition were significantly lower than in both the thick and no-palate conditions, which did not differ from one another. (No differences were found in the no-palate condition over time.) However, an examination of the data for individual speakers at Time 60 revealed that one speaker in particular produced /s/ in the thick palate condition with very little change over time, suggesting that this individual failed to compensate for the perturbation despite the lengthy practice period. In addition, two of the speakers appear to have "overcompensated," producing centroids in the thick condition at Time 60 that were substantially higher than those in the no-palate condition. ${ }^{4}$ Importantly, all speakers displayed the group pattern of lower centroid frequencies in the thin palate condition at the end of the practice period, as at the outset.

With respect to the measure of standard deviation [SD], mean SD measures in all conditions at all time periods are plotted in Fig. 3, illustrating a quasilinear increase in the standard deviation over time. A Time $\times$ Palate ANOVA yielded only a main effect of Time $[F(4,32)=8.552, p$ $<0.001]$, with significant increases in SD measures between Time 0 and Times 45 and 60, as well as between Time 15 and Times 45 and 60, and between Time 30 and Time 60 . The Time $(\mathrm{T} 0, \mathrm{~T} 60) \times$ Palate (no palate, thin, thick) ANOVA comparing no palate and palate conditions again revealed a main effect of Time only $[F(1,8)=7.593, p<0.05]$, in keeping with the pattern described above. One possible interpretation of the SD measure is that it reflects the length of the constriction formed or the involvement of tongue blade (versus the tongue tip) in generating a constriction, with increased SD reflecting a longer (or more laminal) constriction (see, e.g., Stevens, Keywer, and Kawasaki, 1985). Despite the overall group pattern suggesting an increase in SDs over time, though, there was a great deal of variability in the patterns for individual speakers, rendering the group finding questionable.

Mean frication duration measures are plotted in Fig. 4. A Time $\times$ Palate ANOVA yielded only a main effect of Time $[F(4,32)=5.027, p<0.003]$, with significantly longer durations at Time 60 relative to Times 0,15 , and 30; durations at Time 45 and Time 0 also differed significantly. An ANOVA comparing the no palate and palate conditions at Times 0 and 60 only revealed the main effects of Time $[F(2,16$ $=4.945, p<0.05]$ and Palate $[F(1,8)=9.657, p<0.02]$, as well as a Time $\times$ Palate interaction $[F(2,16)=5.375, p$ $<0.02]$. Post hoc analysis of the interaction demonstrated that at Time 0 , fricative duration in the no-palate condition was significantly shorter than in both thin and thick conditions, which did not differ. This pattern was demonstrated by all but a single speaker-the same individual for whom centroid frequencies did not change much over the practice period. At Time 60, no duration differences between palate conditions remained (although there was a good deal of interspeaker variability); further, duration measures were longer at Time 60 than at Time 0 in all conditions, without concomitant increases in overall syllable durations. 


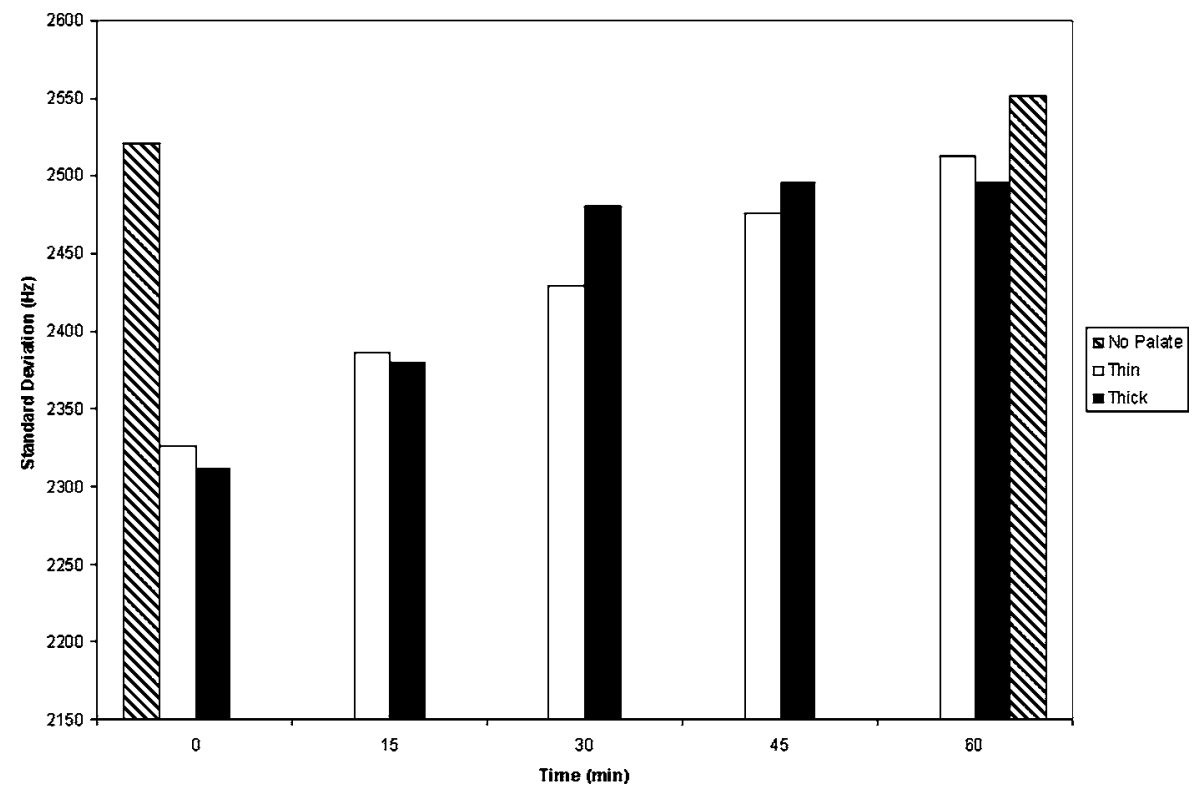

FIG. 3. Mean standard deviation (the second moment of the spectral distribution) at all time periods for the thin and thick palate conditions and at times 0 and $60 \mathrm{~min}$ for the no-palate (NP) condition.

\section{B. EPG analyses}

The average percentage of sensors contacted within the (temporal) region of maximum contact in each condition at each point in time is illustrated graphically in Fig. 5. A Time $\times$ Palate ANOVA yielded a Time $\times$ Palate interaction $[F(4,32)=4.303, p<0.01]$, confirming the patterns apparent in the graph; that is, at Time 0 , contact patterns for both thin and thick palates were comparable (as confirmed by post hoc analyses), whereas at all other times, there was less overall contact in the thin relative to the thick condition. In an examination of individual data, these patterns held for the majority of speakers with a single exception; one speaker exhibited increased contact in the thin relative to the thick palate condition throughout the experiment. ${ }^{5}$ EPG displays for a typical and the exceptional speaker are provided in Figs. 6(a) and 6(b). The contact patterns for the exceptional speaker show the surprisingly increased contact in the thin palate condition, with relatively little change from Time 0 to Time 60.

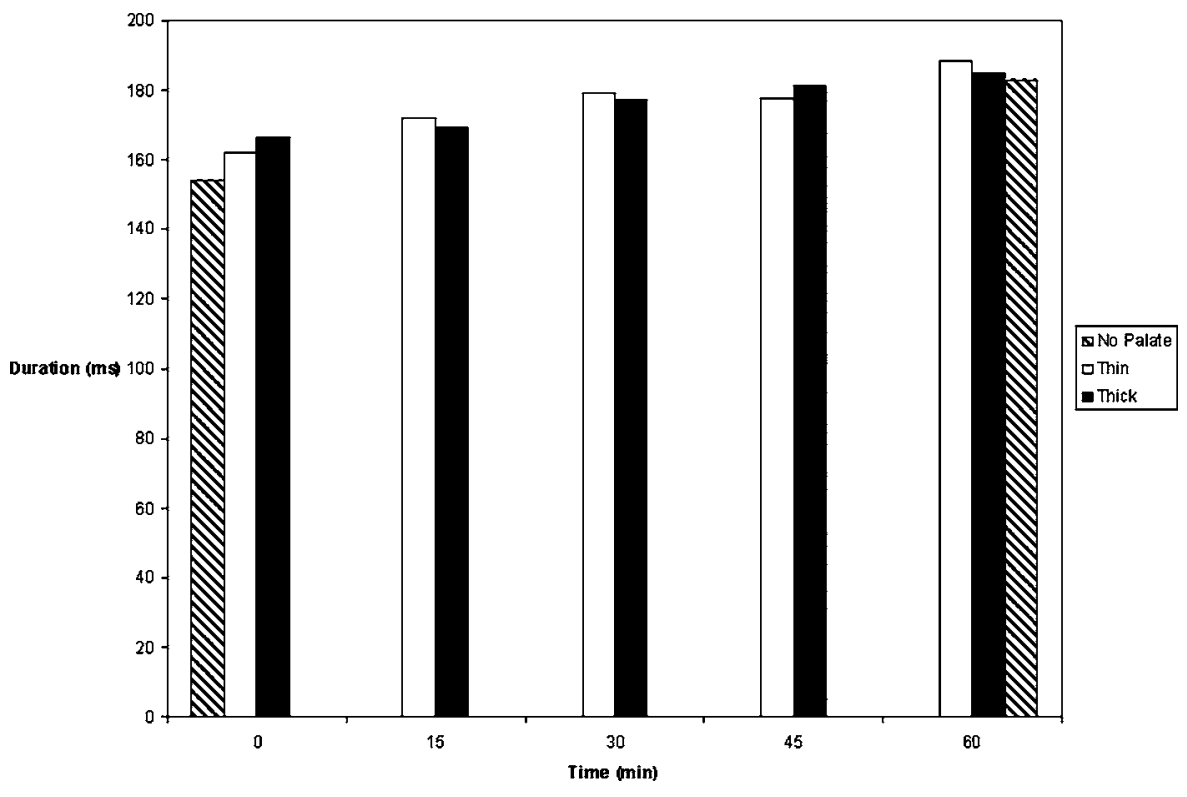

FIG. 4. Mean duration of frication in the /asa/ productions at all time periods for the thin and thick palate conditions, and at times 0 and $60 \mathrm{~min}$ for the no-palate (NP) condition. 


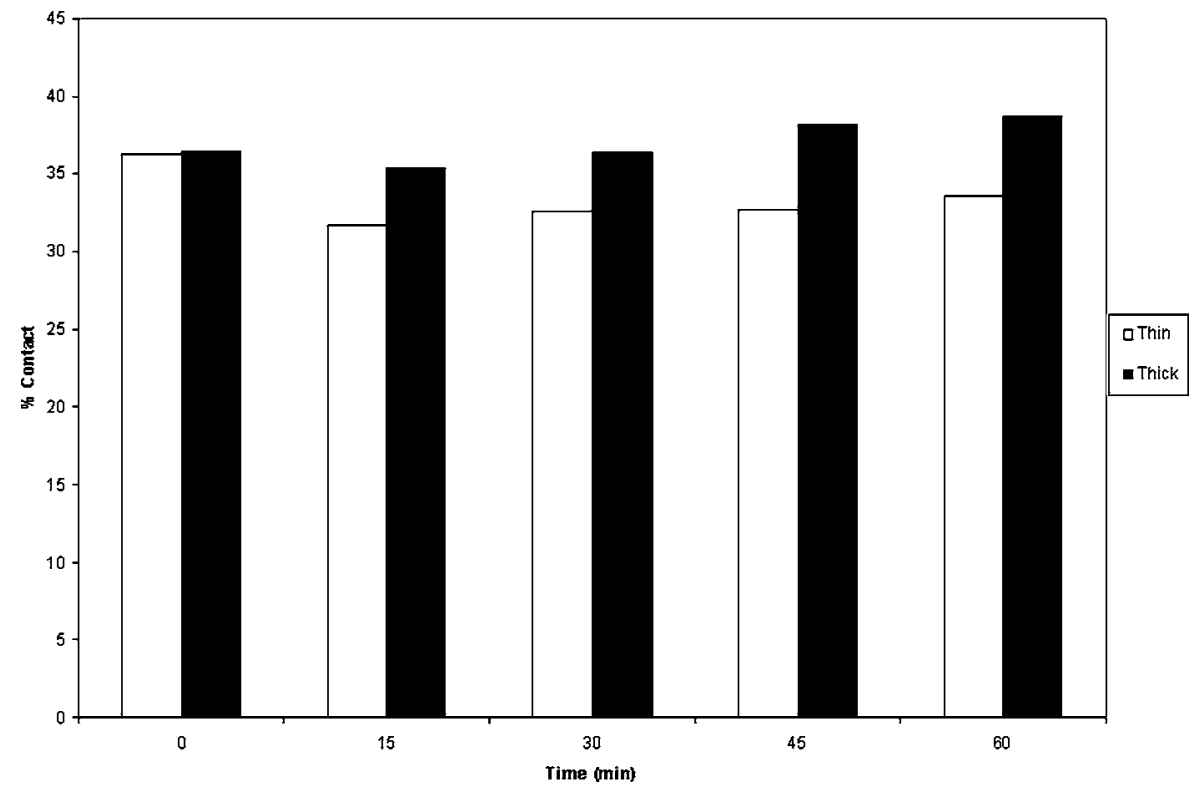

FIG. 5. Mean percentage of sensors contacted during the portion of the /s/ production with maximum tonguepalate contact.

main effect of Palate $[F(1,8)=5.316, p<0.05]$. In examining $\mathrm{CoG}$ indices for individual speakers, all but a single speaker (different from the individual "outlier" noted in the description of maximum contact patterns) generally conformed to the group pattern; this speaker displayed a consistently reversed pattern, with CoG measures in the thin condition lower than in the thick condition at all time intervals.
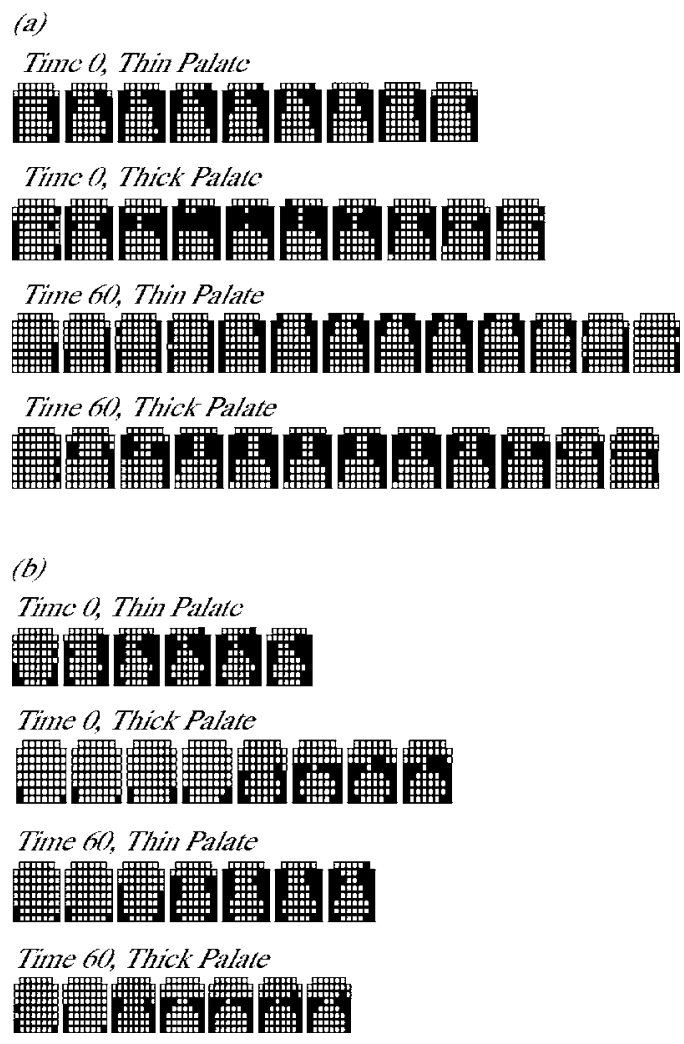

FIG. 6. Contact patterns for exemplar tokens produced by two individual speakers at Times 0 and 60 in both thin and thick palate conditions. The displays represent fricative contact, as defined by the onset and offset of acoustic evidence of frication noise in the waveform; contact patterns are represented in $20 \mathrm{~ms}$ intervals. The first four displays (a) are taken from a speaker who produced typical patterns of contact; the remaining displays (b) are taken from a speaker who produced aberrant patterns.
An analysis of the Laterality index also showed consistently higher values (indicating more lateral contact or a wider groove width) for the thin compared to the thick condition at all time intervals; this pattern held for all individual speakers. The Time $\times$ Palate ANOVA yielded main effects of both Time $[F(4,32)=4.873, p<0.005]$ and Palate $[F(1,8)$ $=169.479, p<0.001]$. Post hoc analysis of the Time main effect revealed significantly higher Laterality index values at Times 15 and 30 relative to Time 0 , collapsed across palate conditions.

Finally, a Time $\times$ Palate ANOVA on the Variability (V) index measures revealed only a Time $\times$ Palate interaction $[F(4,32)=3.844, p<0.02]$. Post hoc analyses showed that, although the patterns of variability in the two palate conditions changed somewhat over time (as illustrated in Fig. 8), none of the differences reached statistical significance.

\section{Perceptual analyses}

Mean perceptual quality ratings in all conditions at all time periods are illustrated in Fig. 9. It is quite clear that initial quality ratings for productions from the thick condition were substantially lower than those for the thin condition. In keeping with the acoustic data, that pattern was reversed at Time 15; after 60 min of practice, quality ratings for both thin and thick conditions were comparable and higher than at Time 0 , but still lower than ratings for stimuli produced with no palate in place. A Time $\times$ Palate ANOVA revealed a main effect of Time $[F(3,27)=26.048, p$ $<0.001]$ and a Time $\times$ Palate interaction $[F(3,27)$ $=55.099, p<0.001]$. Post hoc analysis of the interaction demonstrated that at Time 0 , ratings for the thin palate condition were significantly higher than for the thick palate condition and that pattern was reversed at Time 15. At other time periods, the two palate conditions did not differ. Within the thin palate condition, ratings got significantly worse from Time 0 to Time 15 , whereas within the thick palate condition, ratings improved significantly from Time 0 to Time 15 . In the thin palate condition, from Time 15 to Time 30, ratings 


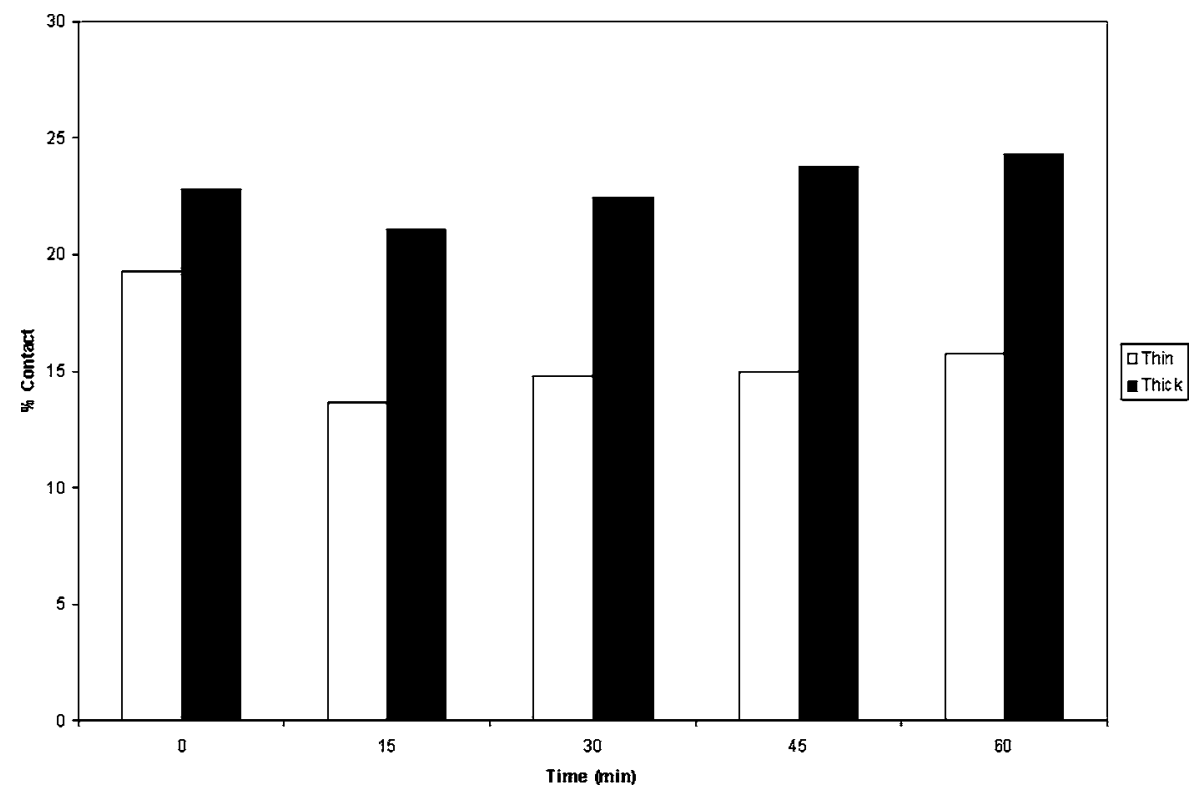

FIG. 7. Percentage of contact in the alveopalatal region (the five most anterior rows of sensors) during the period of maximum contact.

improved significantly. A second ANOVA examining the three palate conditions at Time 60 alone revealed a significant main effect of Palate $[F(2,18)=56.113 . p<0.001]$. Post hoc analysis confirmed significantly higher ratings in the nopalate condition relative to both thick thin palate conditions, which did not differ from one another. An examination of individual data revealed that all listeners exhibited all of the group patterns.

\section{DISCUSSION}

The present investigation was designed to explore speech compensatory processes related to the presence of a rather significant perturbation of oral form using a triangulation of measures involving acoustic, perceptual, and electropalatographic analyses. Electropalatographic electrodes were embedded in both thin and thick palatal appliances to measure compensatory tongue positioning during a period of intense, target-specific practice.

\section{A. Acoustic and perceptual findings}

Target-specific practice appeared to facilitate the development of novel articulatory programs in the face of the perturbation, as revealed by both the acoustic and perceptual profiles. Consistent with our previous findings (Baum and McFarland, 1997; McFarland, Baum and Chabot, 1996) and those of others (Hamlet, Cullison, and Stone, 1979), the thick artificial palate was highly perturbing to /s/ production, with centroid frequencies and quality ratings of /s/ that were significantly lower for this palatal condition in the initial test interval when contrasted with the no-palate and thin palate conditions. (Perturbing effects of the thin palate were also evident, as discussed elsewhere.) Acoustic and perceptual accuracy of the /s/ production with the thick appliance in place increased over the practice interval, and after $60 \mathrm{~min}$ no significant differences were found between the thick and nopalate conditions in /s/ centroid values. This appeared to be related to two factors: an increase in mean centroid values

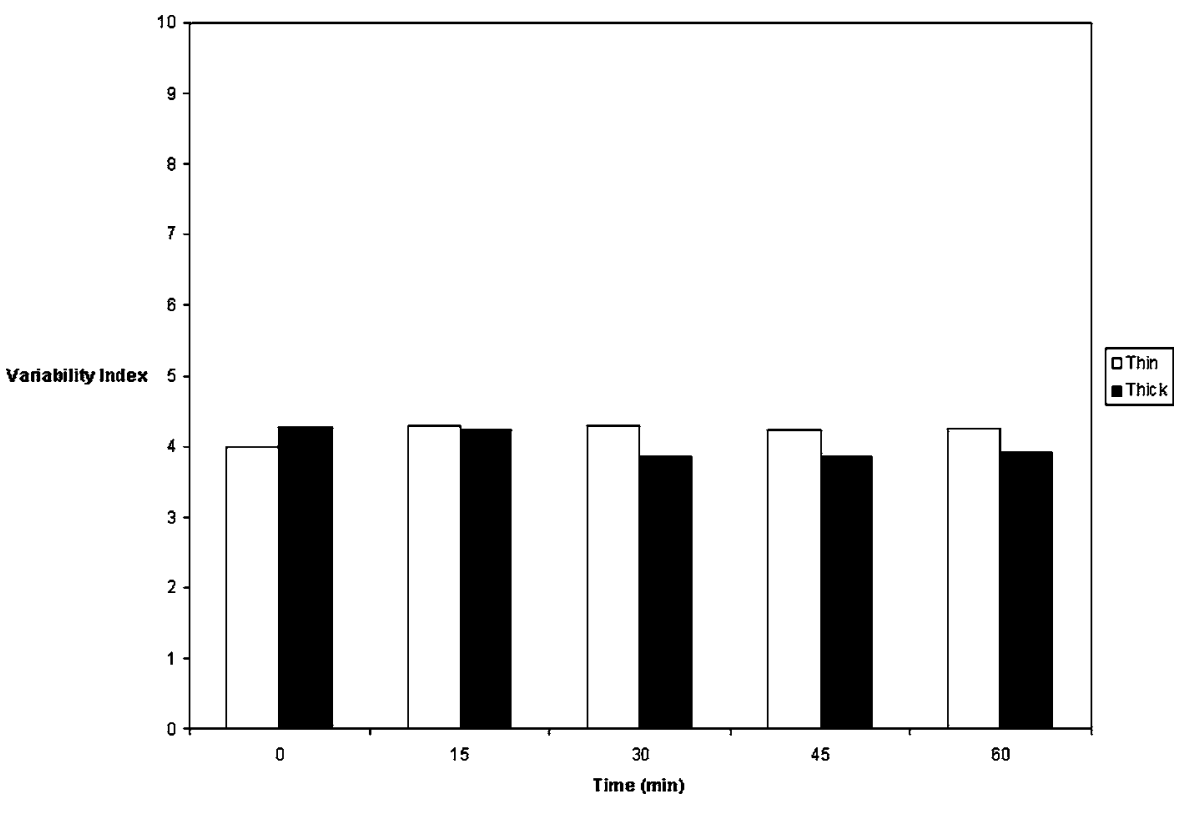

FIG. 8. Mean variability index (ranging from 0-50) during the period of maximum contact. (Higher values indicate greater variability within productions.) 


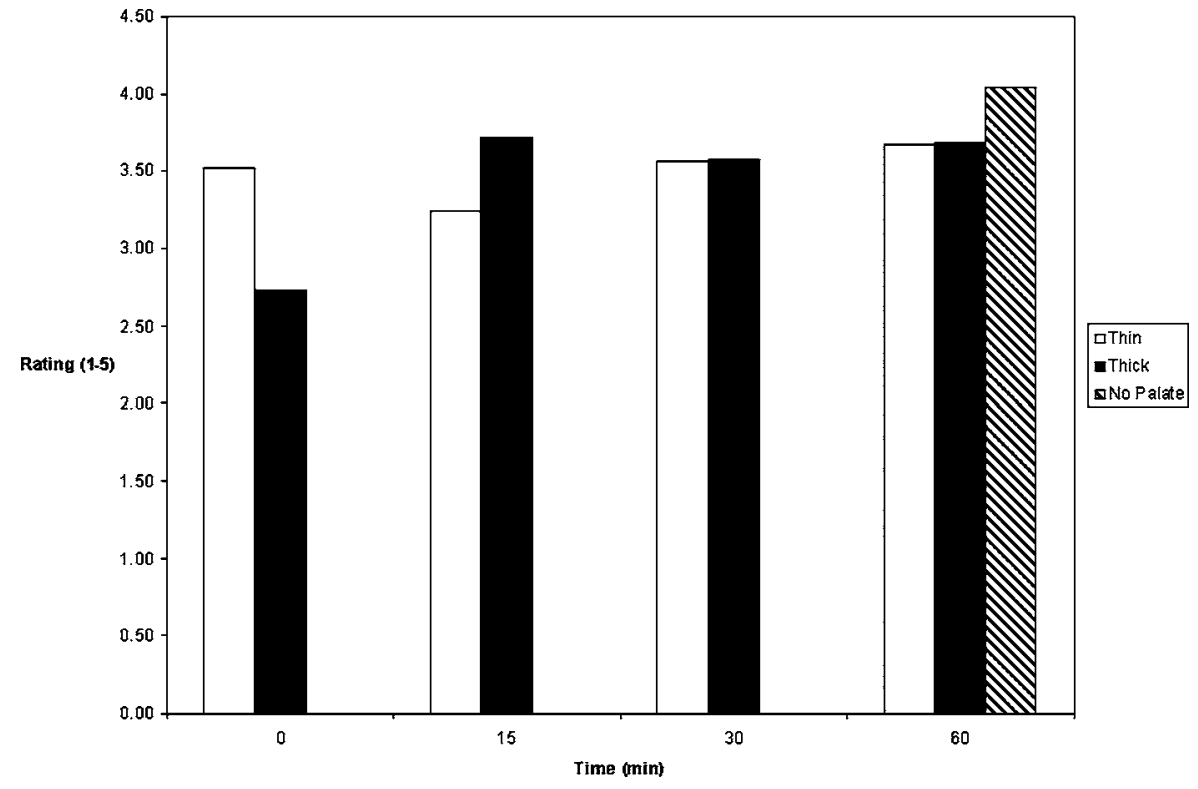

FIG. 9. Mean rating of /s/ quality by listeners, on a scale from 1 (very poor quality /s/) to 5 (very good quality /s/). associated with the thick palate over time, and a (nonsignificant) decrease in centroid values with no palate in place. Similarly, longer /s/ durations were observed across palatal conditions, including the no-palate condition after $60 \mathrm{~min}$ of practice when contrasted to initial measurement periods. The decrease in mean centroid values and increased utterance durations associated with the no-palate condition might be indicative of the overall recalibration of the articulatory profiles associated with /s/ production and distributed effects of the novel articulatory profiles appropriate to the change in oral form associated with the thick palate. In other words, these changes in production with no palate in place may have represented negative aftereffects of the target-specific practice with the thick palate in place. Additional evidence of negative aftereffects comes from the thin palate data. After 15 min of practice with the thick appliance in place, mean centroid values and quality ratings were lower for the thin palate than those computed during the initial measurement period. Negative aftereffects are indicative of the development of new speech articulatory profiles that are appropriate for the change in oral form and function (Jones and Munhall, 2000). Similar effects have been observed in other motor systems, and have been particularly well studied in visualmotor control (e.g., van der Kamp, Bennett, Savelsbergh, and Davids, 1999) and gait control systems (e.g., Anstis, 1995). The fact that such effects can be observed in compensatory speech posturing suggests that similar motor control processes are operating in rather diverse sensorimotor systems.

\section{B. EPG findings}

Several inter-related measures of tongue contact were made that supplemented the acoustic and perceptual results highlighted above. Increased maximum contact, posterior tongue positioning, and more medial contact patterns were observed for the thick as contrasted to the thin palatal appliance, although individual differences were apparent. These tongue contact patterns indicate potential tongue overshoot and flattening in response to the buildup of acrylic over the alveolar ridge (see Narayanan et al., 1995; Stone et al., 1992). In turn, there is likely to be a narrower and decreased depth of tongue groove due to the presence of the buildup of acrylic over the alveolar ridge. This in turn seems likely to have influenced turbulence generation and consonant spectral characteristics. Consequently, /s/ production under these conditions yielded lower centroid frequencies and lower quality ratings. Previous combined EPG and ultrasound studies have revealed that $/ \mathrm{s} /$ is normally associated with relatively marginal lateral lingual palatal contact (when contrasted with / $/$ /, for example) (Stone et al., 1992). Increased medial tongue contact under conditions of perturbation may be related to mechanical compression against the acrylic buildup (Stone et al., 1992). It might also represent a compensatory posturing of the tongue in response to the change in oral form. Previous kinematic studies have revealed the relatively marginal lateral tongue palate contact is importantly involved in the generation of "lingualpalatal bracing" necessary for appropriate tongue grooving (Flege et al., 1988; Stone et al., 1992). Perhaps increased medial contact allowed for compensatory changes in tongue shape and the redirection of the airstream, which resulted in overall improvements in fricative spectral characteristics and quality over the course of the practice interval. That is, there may have been other changes in tongue shape-for example, midsagittal groove dimensions (Fitzpatrick and Ní Chasaide, 2002) - supported by the compensatory tongue positioning, that would not have been revealed by our EPG measures and would require direct assessments of tongue shape related to groove configuration such as those provided by ultrasound, MRI or EMA (e.g., Cheng, Goozée, and Murdoch, 2005; Fitzpatrick and Ní Chasaide, 2002; Hoole, Nguyen-Trong, and Hardcastle, 1993; Narayanan et al., 1995; Stone et al., 1992). Decreased tongue contact was observed with the thin palatal appliance over time, suggesting rather rapid compensation to this relatively small perturbation to an oralarticulatory structure. Similar findings of rapid compensation were observed in our previous investigations of the thin versus thick appliances using non-EPG measurement proce- 
dures (McFarland, Baum, and Chabot, 1996). The subtle changes observed in the acoustic and perceptual data indicating negative aftereffects on the thin palate condition were less apparent in the EPG patterns, again arguing for an additional measurement of tongue shape or movement.

Overall, the combined measurement systems used in the present investigation revealed evidence of the development of compensatory articulatory programs after an intense, but relatively short duration target-specific practice. We have previously suggested that such compensation may represent a sensorimotor recalibration and the development of novel speech motor programming appropriate for the change in oral form and function (Baum and McFarland, 1997). This interpretation is supported by additional evidence of negative aftereffects, where the new articulatory profiles were apparently applied to nonperturbed productions and resulted in disrupted speech production, even without the palate in place. It seems logical to assume that sensory feedback would be relatively crucial in the development of these novel programs and may include information related to tongue positioning/tongue contact as well as the acoustic (and perceptual) consequences of the novel articulatory gestures. The fact that speech acoustic parameters associated with /s/ production improved significantly during the practice interval, while tongue contact patterns remained relatively constant, may be interpreted as suggesting that speakers are relying on auditory or perceptual goals as contrasted to those related to kinesthetic feedback. Alternatively, it could be argued that kinesthetic feedback was involved in modulating articulatory gestures and in particular groove shape or tongue configuration, but these were not apparent to our levels of analysis.

The fact that learning appears to occur in a relatively brief period with target-specific practice, despite the highly perturbing nature of the oral articulatory appliance, supports the utility of this type of experimental paradigm to explore these types of fundamental aspects of speech production. As in our previous investigations, there was a good deal of individual variability in the degree of adaptation, with some speakers exhibiting relatively quick compensatory adjustments and others (at least one) who appeared to show no evidence of compensation to the presence of the artificial palates, even after the $1 \mathrm{~h}$ practice interval. In our previous investigations, this one-hour practice interval was sufficient to allow at least some speakers to adapt to the presence of an artificial palate (Baum and McFarland, 1997; 2000). Several factors may account for these individual differences, including differences in palatal morphology, tongue motility, and speakers' perceptual sensitivity. With regard to the latter factor, Perkell and colleagues (2004) have recently provided data suggesting that the production strategies utilized by individual speakers depend to a great extent on their discriminative sensitivity for the specific speech contrast under investigation. In future studies, it will be interesting to examine the perceptual abilities of individual speakers in relation to their speech adaptation patterns as well as potential anatomical constraints on compensatory abilities. Clearly, individual compensatory strategies are of great interest in understanding motor control strategies related to the development and perhaps refinement of oral articulatory profiles (Flege et al.,
1988). The fact that there are apparently sometimes rather large differences in articulatory contact and configuration in response to oral perturbation might have important clinical implications as well, particularly given the fact that EPG measures are often used to remediate deviant vocal tract form and function such as that related to velopharyngeal insufficiency. Comparing subjects to idealized "normal" contact patterns may not be the most appropriate technique and may in fact undermine individual compensatory strategies. In fact, recent EPG investigations involving remediation of speech-language disorders have emphasized the importance of considering individual characteristics in speech production abilities as a predictive function influencing treatment success (e.g., Carter and Edwards, 2004; Gibbon, 2004).

The findings of the present investigation confirm and extend those of our previous investigations (Baum and McFarland, 1997; McFarland, Baum, and Chabot, 1996), indicating substantial flexibility within the speech production system, permitting the majority of speakers to adapt to a significant oral-articulatory perturbation with (relatively) minimal practice. Interestingly, in contrast to the acoustic and perceptual results, the EPG data revealed few changes in tongue-palate contact over time, suggesting that compensatory adjustments for /s/ production were made by tongue shape changes in regions that did not contact the palate. Future investigations using methods that capture tongue contour more directly will elucidate precisely what those adaptive strategies may be and how they may vary across individual speakers.

\section{ACKNOWLEDGMENTS}

This work was supported by a grant from the Natural Sciences \& Engineering Research Council of Canada (NSERC) to the second author, and a scholarship from the Fonds de la Recherche en Santé du Québec (FRSQ) to the first author. We are indebted to Meg Grant for her assistance in data analysis and manuscript preparation.

\footnotetext{
${ }^{1}$ The thin palate presumably reflected an unperturbed state and was required to record EPG contact patterns.

${ }^{2}$ Prior to any experimental recording, subjects inserted the thin palate and produced the phrase "I said 'ahh' doctor" five times. This was done to ensure that during normal vowel production for these speakers, no electrodes were consistently contacted; had any electrodes been contacted during /a/, they would have been removed from analyses of contact patterns for $/ \mathrm{s} /$. This proved unnecessary.

${ }^{3}$ Any production produced with no palate in place at Time 0 could be included unless precluded by noise in the recording or hesitation on the part of the speaker

${ }^{4}$ Importantly, means computed excluding these three individuals do not differ substantially from those for the group as a whole, as illustrated in the figure. In the no-palate condition, with the exclusion of these speakers, mean centroids were 4439 and $4189 \mathrm{~Hz}$ at Times 0 and 60, respectively; in the thin palate condition, values were 3893 and $3812 \mathrm{~Hz}$ at Times 0 and 60; in the thick palate condition, centroids were 3723 and $4172 \mathrm{~Hz}$ at Times 0 and 60 , excluding these three speakers.

${ }^{5}$ Interestingly, this was not the same speaker who appeared to be an "outlier" based on the acoustic analyses.
}

Aasland, W., Baum, S., and McFarland, D. (2004a). "Adaptation to structural modifications of the vocal tract during speech: Electropalatographic and acoustic measures," paper presented at From Sound to Sense: Fifty + Years of Discoveries in Speech Communication, Cambridge, MA. 
Aasland, W., Baum, S., and McFarland, D. (2004b). "Adaptation to structural modifications of the human vocal tract during speech: Electropalatographic measures," paper presented at Acoustical Society of America, New York, NY.

Anstis, S. (1995). "Aftereffects from jogging," Exp. Brain Res. 103, 476478.

Baum, S., and McFarland, D. (1997). "The development of speech adaptation to an artificial palate," J. Acoust. Soc. Am. 102, 2353-2359.

Baum, S., and McFarland, D. (2000). "Individual differences in speech adaptation to an artificial palate," J. Acoust. Soc. Am. 107, 3572-3575.

Baum, S., McFarland, D., and Diab, M. (1996). "Compensation to articulatory perturbation: Perceptual data," J. Acoust. Soc. Am. 99, 3791-3794.

Carter, P., and Edwards, S. (2004). "EPG therapy for children with longstanding speech disorders: predictions and outcomes," Clin. Ling. \& Phon. 18, 359-372.

Cheng, H. Y., Goozée, J. V., and Murdoch, B. E. (2005). "Analysis of articulatory dynamics in dysarthria following brain injury in childhood using electromagnetic articulography and electropalatography," J. Med. Sp.-Lang. Path 13, 15-35.

Fitzpatrick, L. and Ní Chasaide, A. (2002). "Estimating lingual constriction location in high vowels: a comparison of EMA- and EPG-based measures," J. Phonetics 30, 397-415.

Flege, J. E., Fletcher, S. G., and Homiedan, A. (1988). "Compensating for /s/ and / / production: Palatographic, acoustic and perceptual data," J. Acoust. Soc. Am. 83, 212-228.

Fletcher, S. G., and Newman, D. G. (1991). "[s] and [ $\left.\int\right]$ as a function of linguapalatal contact place and sibilant groove width," J. Acoust. Soc. Am. 89, 850-858.

Fletcher, S. G., McCutcheon, M. J., and Wolf, M. B. (1975). "Dynamic palatometry," J. Speech Hear. Res. 18, 812-819.

Gibbon, F. E. (2004). "Abnormal patterns of tongue-palate contact in the speech of individuals with cleft palate," Clin. Ling. \& Phon. 18, 285-311.

Gibbon, F. E., and Wood, S. E. (2003). "Using electropalatography (EPG) to diagnose and treat articulation disorders associated with mild cerebral palsy: a case study," Clin. Ling. \& Phon. 17, 365-374.

Goozée, J. V., Murdoch, B. E., and Theodoros, D. (2003). Electropalatographic assessment of tongue-to-palate contacts exhibited in dysarthria following traumatic brain injury: Spatial characteristics," J. Med. Sp. Lang. Path. 11, 115-129.

Hamlet, S., Cullison, B., and Stone, M. (1979). "Physiological control of sibilant duration: Insights afforded by speech compensation to dental prostheses," J. Acoust. Soc. Am. 65, 1276-1285.
Hamlet, S., Stone, M., and McCarty, T. (1978). "Conditioning prostheses viewed from the standpoint of speech adaptation," J. Prosthet. Dent. 40, 60-66.

Hoole, P., Nguyen-Trong, and Hardcastle, W. (1993). "A comparative investigation of coarticulation in fricatives: Electropalatographic, electromagnetic, and acoustic data," Lang Speech 36, 235-260.

Jones, J. A., and Munhall, K. G. (2000). "Perceptual calibration of F0 production: evidence from feedback perturbation," J. Acoust. Soc. Am. 108, 1246-1251.

Löfqvist, A. (1997). "Theories and models of speech production," in The Handbook of Phonetic Sciences, edited by W. J. Hardcastle and J. Laver (Blackwell Publishers, Inc., Cambridge, MA).

McFarland, D., and Baum, S. (1995). "Incomplete compensation to articulatory perturbation,” J. Acoust. Soc. Am. 97, 1865-1873.

McFarland, D., Baum, S., and Chabot, C. (1996). "Speech compensation to structural modifications of the oral cavity," J. Acoust. Soc. Am. 100, 1093-1104

Narayanan, S., Alwan, A., and Haker, K. (1995). "An articulatory study of fricative consonants through magnetic resonance imaging," J. Acoust. Soc. Am. 98, 1325-1347.

Parsloe, R. (1998). "Use of speech pattern audiometer and the electropalatograph to explore the speech production/perception relationship in a profoundly deaf child," Int. J. Lang Commun. Disord. 33, 109-121.

Perkell, J., Matthies, M., Tiede, M., Lane, H., Zandipour, M., Marrone, N., Stockmann, E., and Guenther, F. (2004). "The distinctiveness of speakers' $/ \mathrm{s} /-/ \mathrm{s} /$ contrast is related to their auditory discrimination and use of an articulatory saturation effect," J. Speech Lang. Hear. Res. 47, 1259-1269. Stevens, K. (1998). Acoustic Phonetics (MIT Press, Cambridge, MA).

Stevens, K., Keywer, S., and Kawasaki, H. (1985). "Toward a phonetic and phonological theory of redundant features," in Invariance and Variability in Speech Processes, edited by J. Perkell and D. Klatt (Lawrence Erlbaum Associates, Hillsdale, NJ).

Stone, M., Faber, A., Raphael, L. J., and Shawker, T. H. (1992). "Crosssectional tongue shape and linguopalatal contact patterns in [s], [], and [1]," J. Phonetics 20, 253-270.

Tremblay, S., Shiller, D. M., and Ostry, D. J. (2003). "Somatosensory basis of speech production," Nature 423, 866-869.

van der Kamp, J., Bennett, S. J., Savelsbergh, G. J., and Davids, K. (1999). "Timing a one-handed catch. II. Adaptation to telestereoscopic viewing," Exp. Brain Res. 129, 369-377.

Wrench, A. (2003). Articulate Assistant (Articulate Instruments Ltd., Edinburgh). 